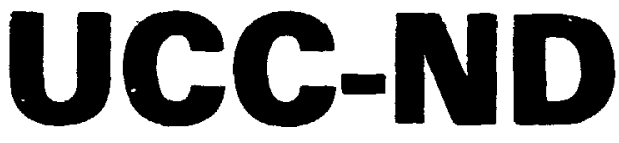

NUCLEAR DIVISION ,
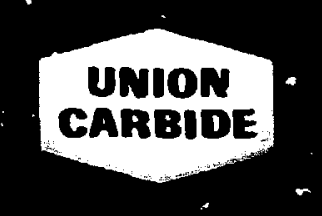

4

DPEAAIFO BY

UNION CARBIOF COGRPJIFATION

FOR THE UNITSO STAIS:

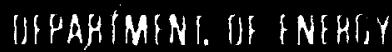

DR. 324

ORNL/CSD-45

\section{Observations on Risk Analysis}

W. A. Thompson. Jr.

\title{
MSTER
}


rontract No. W-7405-eng-26

COMPUTER SCIENCES DIVISION

OBSERVATIONS ON RISK ANALYSIS

(with special reference to energy related problems)

W. A. Thompson, Jr.

Sonsor: D. A. Gardiner

Originator: W. A. Thompson, Jr.

\section{Dace Published - November 1979}
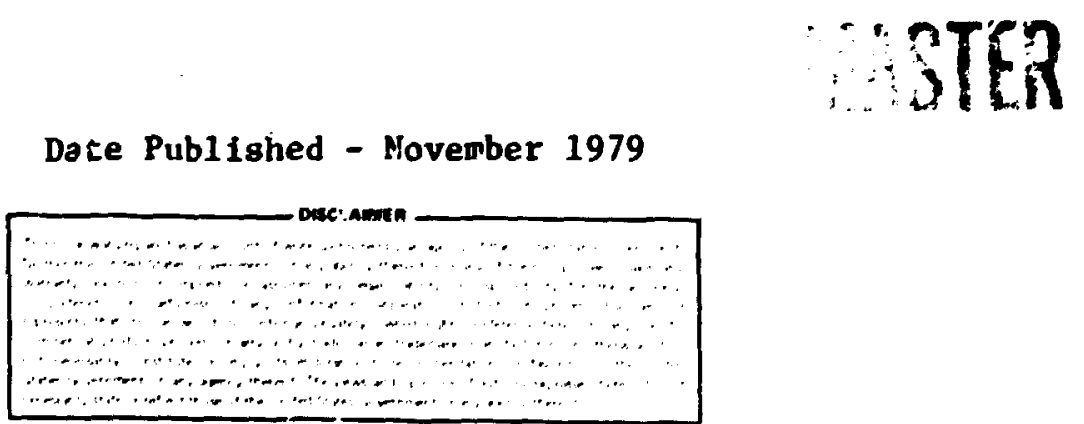

UNION CARBIDE CORPORATION, NUCLRAR DIVISION operating the

Oak Ridge Gaseous Diffusion Plant Oak Ridge National Laboratory Oak Ridge Y-12 Plant for the DEPARTMENT OP ENERCY 
ABSTRACT

This paper briefly describes HASH 1400 and the Lewis report. It attempts to define basic concepts such as risk and risk analysis, common mode failure, and rare event. Severai prubabilistic models which go beyond the WASH 1400 methodology are introduced; the common characteristic of these models is that ihey recognize explicitly that risk analysis is time dependent whereas WASH 1400 takes a "per demand failure rate" approach which obscures the important fact that accidents are time related. Further, the presentation of a realistic risk analysis should recognize that there are variuus risks which compete with one another for the lives of the inoividuals at risk. A way of doing this is sugyested. 
"But in this world noching can be sajto be vertain. tares." - Benjamin Franklin

\section{IHT SCOUCTIA}

\subsection{Applications.}

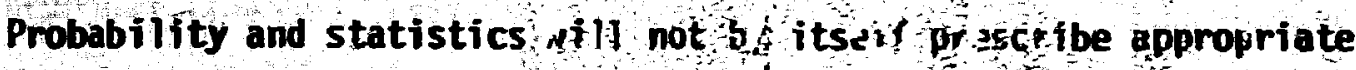
energy policy; decisions will he re to be uje sy sper iaftsts in these. natters, but probability and stotistice can lef siustantial help to the energy specialist in maki 1 ars decisiot If following examples illustrate the kinds of applicitions whech articipate.

When a new potentialiy dargisous tofincligical change such as an airport, highway, or nuclear power plar is rieztemplated for a community, a rational approach will be ts weig the dyarsages and disacvantages before making a decision. One of the diskdvantages is that arcidents may cause fatalities and injiny. Hev lat we quantify this risk?

How can we compare the risks is aciated with alternative methods of energy production? For eyampl:, hou does the risk of mine disaster and health damage associated wit coal ming compare with the nuclear generator risk?

How do the risks of alternitive uethods of nucloar waste disposal compare with one another? What are the risks astiated with transfrorting spent fuel to a suitable disposis area?. I- it safe to leave uranium mine tailings unprocessed? What is the danger that underground storage will contaminate drinking waler owe to the mobfllty of subsurface water? 
Now, switching to a more fundamental example, consider that organisms are exposed to "doses" alleged to have detrimental effect; the doses may, for example, be of radiation or a chemical substance. The organism may or may not "respond," for example, by developing a cancer.

Determination of the true relation between response and dose is an important but difficult task. It is important because it is a basic issue in assessing the risk of an activity ror safety or health, and therefore, it is of fundamental concern for the government regulation of industrial activity, nuclear generators for instance. It is difficult because it is interdisciplinary, bordering on biology, medicine, genetics, physics, and chemistry; it has a strong statistical component. Data is hard to obtain and frequently it is only indirectly to the point.

Thus, as these examples suggest, with increased population density, environmental awareress and technological intensity the government in general and the Department of Energy in particular has become more involved in risk assessment and regulation. These are all difficult problems but they are not, as is sometimes thought, entirely new problems. "... the use of quantitative techniques in decision making associated with risk is still in its early stages and is highly formative," WASH 14C2. (1975). This is only partly true; risk analysis has a probabilistic and statistical component going back at least to Daniel Bernoulli.

\subsection{What is risk analysis (RA)?}

Webster (1976) gives as the first meaning of risk: "the possibility of loss, injury, disadvantage, or destruction: CONTli:ieNCY, DANGER, PERIL, THREAT." "Risk is the potential fC: realization of unwanted, 
negative consequences of an event," Rove (1977). In the statistical literature the word risk is not used in the above sense. There are several statistical meanings of risk identified with actuarial risk theory, decision theory and competing risk theory. We return to these in Section 4.

Formally, risk of the kind being discussed in RA, may simply be a random variable. In the background there is an activity to be engaged in and $\geq$ collection of events wich could result. Which event does result is subject to chance. In this context, events are usually thought of as being unpleasant but they need not be. Associated with each event there is a consequence of the event occurring. If the consequence is numerical and if by risk we mean the uncertain consequence before the activity is engaged in, then in the language of probability, risk is a random variable. The events will have probabilities which can be transferred to the consequences to obtain the probability distribution of the risk. Of course, risks and random variables can be vector vaiued.

A special motivation for $R A$ is the need for assessing the impact of technological innovation on so-ietal safety through aizeration of the environment. The purposes of RA are to choose between alternarive actions or policies and to judge whether an activity exceeds minimum stancards of acceptable risk. Thus RA may help in formulating new laws or regulations, assessing existing regulatory requirements, eva 1wating new designs, indicating needed repairs, formulating safety research and inspection priorities, developing of licensing guidelines and dealing with general safety issues. In the design task we may attempt to prescribe what process parameter values will produce the desired system safety characteristics with a prescribed probability. 
RA i) identifies the potential outcomes, both good and bad, of actions or policies $i$ ! quantifies as far as possible the consequences of the outcomes including who will bear the burdens and wo the benefits iii) estimates the probabilities of the consequences and iv) weighs the good against the bad.

\section{REACTOR SAFETY STUDY (WASH 1400)}

WASH 1400 (i975), sometimes called the Rasmussen report, is the state of the risk analysis art, at least for energy related problens. The purpose of the report is indicated by $i t s$ subtitle: "An Assessment of Accident Risks in U.S. Commercial Nuclear POwE. Plants." Till HASH 1400, it was not clear kow one might approach such problems. The report consists of three parts, i) a popular, non-technical executive sumary of 12 pages which gives the general impression that, all things considered, nuclear power is really quite safe, ii) a technical main report cf 198 pages, and $i$ ii) a cubic foot of appendices indicating the vast amount of detail wich went into the preparation of the report.

It is worthwhile for us to suggest the flavor of the WASH 1400 methodology; "The risk determination was divided into three major tasks.... Task I includes the identification of potential accidents and the quantification of both the probability and magnitude of the associated radioactive releases to the environment.... Task II uses the radioactive source term defined in Task I and calculates how the radioactivity is distributed in the environment and what effects it has on public health and property.... Task III combines the consequences calculated in Task II, weighted by their respective probabilities to produce the overall 
risk from potential nuclear accidents. To give some perspective to these results, they are compared to a variety of non-nuclear risks. "In Section 4. we suggest a way to make this comparison more complete and formal.

With regard to Task $I$, accident seq:Iences arr identified by means of logic diagrams called event trees. "The starting point for the development of an event tree is the event (failure) that initiates a potential accident situation." A typical initiating event is a pipe break which may cause a loss of coslant accident (LOCA). Initiating events must be identified by the analyst using technical information and engineering judgement. A necessary reakness of this and presumably other methods appears at this point: there is no way of being sure that an important initiating event has not been overlooked. An event tree consists of an initiating event and the success or failure response of each of the applicable engineered safety features.

"After identifying the accident sequences, we must assign the probability of occurrence of each engineered-safety-system failure in the particular sequence. Since no commercial reactor has ever experienced these failures, no empirical data on which to base estimates of system-failure rates exist. Thus it is necessary to use techniques that generate system failure rates from failures of components and subsystems. Fault-tree methodologies, developed by the Department of Defense and NASA to identify system design flaws, are extended to derive probability estimates for both the failure of individual nuclear safety systems and the accident sequences of interest. The failure rates of components and sulsystems are determined from industrial experience with products similar to those either in nuclear service or in related usz in other industries." Rasmussen (1974).

The following problem is typical and suggestive of the Fault-tree methodology computation: On demand, an engineering safety feature requires both $A-C$ and $D-C$ current to operate. The probabilities of loss of 1) all $D-C$ power 2) off-site A-C power and 3) on-site A-C power are $P_{1}, P_{2}$, and $P_{3}$ respectively. What is the probability that the safety feature fails 
to operate due to loss of power? Answer: $1-\left(1-p_{1}\right)\left(1-p_{2} p_{3}\right)$ if events are independent.

Figure 1, taken from Rasmussen (1974), indicates the nature of a typical filled out event tree. Note i) that the tree begins with the initiating event "pipe break"; ii) it is simplified in that only seven of the $2^{4}$ possibilities need be considered; for example, if electric power fails then none of the engineering safety features will function; iii) $\mathrm{P}_{2}$ is the answer the just previously considered problem;iv) release categary consequences with their probabilities are the output of the event tree; v) probabilities are approximated by omitting all 1 - $P_{i}$ terms since $P_{i}$ is small.

\section{Common mode failures}

From WASH 1400: "The most uncertain area in the study (is) related to whether potential common mode failures or dependent failures, could be properly identified."... "In a simplified way, common mode failures can be thought of as multiple failures caused by a single event or failure e.g. the same environmental condition."

One thought which might help in the treatment of dependent failures is that the list of initiating events might be enlarged and redefined to eliminate dependencies. Thus suppose that an analysis treats the two initiating events $P=\{$ pipe break $\}$ and $T=\{$ transfent $\}$ and that we want to incorporate $F=\{$ flood $\}$ and $H=\{$ human error $\}$. Define the new initiating events $F, H \cap \bar{F}, P \cap \bar{F} \cap \bar{H}$, and $T \cap \bar{F} \cap \bar{H}$ and prepare four event trees for these initiating events. Cne branch of the first event tree would consider that a fluod causes a pipe break and there is a hunian error in the attempted repair. 


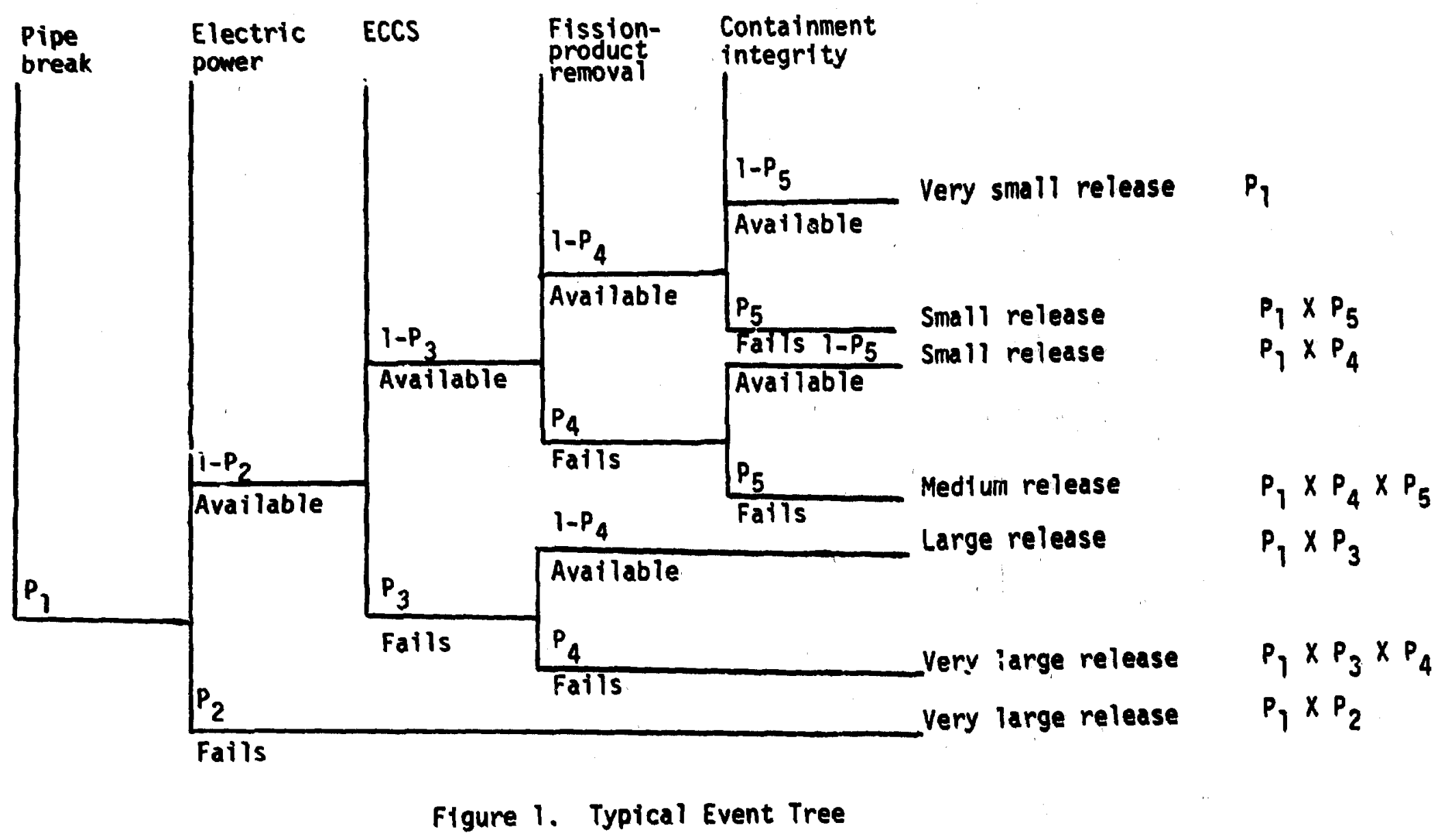


The Lewis et al. report (1978) criticizes WASH 1400. They point tinadequacies in statistical methorology, particularly the uncritical use of the log-normal distribution. They question metner the quality and availability of the data base and the completeness of the study, vast as it is, is adequate to support the executive sumary conclusion. They seem to be saying that the siudy is a good try out the uncertainty of its conclusions is understated and toc substantial to support a decision on the safety of nuclear power. Shortly thereafter the Nuclear Regulatory Commission repudiated the conclusions of the executive sumary, just in time for Three Mile Island.

\section{DETERHINATION OF PROBABİLITIES}

Ideally we should estimate the probabilities of the events in a risk analysis by direct observation using the methods of Demography and Statistical Reliability, see Spiegelman (1976) and Mann et al. (1974). However, the events will often be catastrophic and, fortunately for the individuals at risk, quite rare. This is however unfortunate for the analyst as he will have little data to work with.

"The breaking up of a rare rvent into a series of more likely events is a basic principle of the event tree and fault tree techniques..."

WASH 1400 uses a per-demand failure rate approach synthesizing system failure rates by fault-tree and event-tree methodology into more readily avaflable component rates. Thus in our figure 1 and its discussion the $P_{i}$ 's were (conditiona1) probabilities and we multiplfed them together to obtain the probability of a consequence. But all is not as it seems. Lteberman (1976) complains, rightly, 
about the use of probabilities greater than one. For example, on page 167 of WASH 1400 we find that $P_{\text {IE }}$.hich was previously the probability of an initiating event, is now 10 transients per reactor year but we still multiply as in Figure 1 to obtain the probability of a consequence.

The answer here is correct under plausible ass mptions. Let's simplify the MASH 1400 problem to the follouing: Suppose that transients occur at random in operating time at the rate of 10 ier reactor year and that when a transient occurs the conditional probability that the safety system does not function and an accident results is $10^{-10}(=q=1-p)$. The probability of one or more accidents per reactor year is then

$$
\begin{aligned}
i \stackrel{\infty}{i}\left(1-p^{i}\right) 10^{i} & \exp (-10) / i !=1-\exp (-i 0 q) \\
& =10 . q=10^{-9} .
\end{aligned}
$$

We still multiply things together; the error was one of exposition and failure to state assumptions.

The Lewis report suggests that in some cases it may be possible to do better, they refer to a review of boiling water reactor scram fallures. The correct basis for that review amouilts to the following:

\section{Safety System Effectiveness Problem.}

The rate of reaction in an atomic power plant is controlled at a safe level by the insertion of contro rods. The times at which control is needed (called transients) are assimed to be a Poisson process with rate $\mu$ per year. Failure times of the control system is assumed Poisson with rate $\theta$ per year. Transients and control system faliures are assumed independent. 
Wi thin tine $T$, no transient will occur when the safety system is innperable if, first, the safety system operates for time $T$, or secoild, the earliest safety system failure occurs at time $t$ but no transient occuis before the end of the interval. Hence the probability of this event is:

$$
\begin{aligned}
P(T) & =\exp (-\theta T)+\int_{0}^{T} \theta \exp (-\theta t) \cdot \exp [-\mu(T-i)] d t \\
& =[\mu \exp (-\theta T i-\theta \exp (-i T)] /(\mu-\theta), \mu \neq \theta
\end{aligned}
$$

Next assume that failure of the sufety control system is not detected insediateiy but only at regularly scheduled tests of which there are $N$ per year. The yearly probability that a transient will occur at a time when the safety system is inoperable is $1-\left[P\left(N^{-1}\right)\right]^{N}$. Th. Is we have a second entirely different basis for computing the anrual probability of an accident.

The Lewis report seems to be suggesting that in some instances there may be a physical theory which can be used to obtain becter probability estimates. Indeed this has to offer the best long term possibility for improvement; we sifmply need to know riore about the physics of failure. This includes subjects like reliability, strength of materials, fracture mechanics, corresion, wear, etc.

The above safety system example illustrates a second quite general point. Risk analysis is really time dependent. The time ordering of everts is often important.

Do rare events play a special role? Are theic things which we can do to analyse rare events which are not, valif for arbitrary events? Yes, there are at least two ways of thinking which are particularly applicable to rare events. The first of these is extrume value theory advocated in the present application by Starr, Rudman and Whipple (1976). 
As an example, consider the determination of the dose-response relation discuss:d in our introduction. Traditional methods of fitting the dose-response curve are probit and logit analysis, Finney (1964). Biologically motivated "hit theory" models lead to different distributions, see Turner (1975). Thompson and Funderlic (1979) obtain a Heibull relation as a consequence of the following threshold model: 1) An organism might or might not respond after exposure to dose $X$, 2) the organism has a large number of critical targets, 3) each target has a dose threshold which if exceeded will cause $d$ response in that target, 4) a single target respanse results in organism response, and 5) thresholds of targets are randomly chosen from some distributions. Thus the organism responds if the dose exceeds the minimum target threshold and a minimal extreme value distribution is called for. Several sets of data are fitted and it turns out that the Weibull does as well as the traditional methods. The parameters of the Heibull have nice interpretations.

A second special role is suggested by the fact that the Poisson distribution is sometimes referred to as the law of rare events. The following is a version most useful for risk analysis.

\section{Towards an Algebra of Rare Events.}

To be concrete suppose that there are a large number, $n$, of different kinds of accidents which can occur independently of one another at an industrial plant and that we are interested in the summary process of accidents. "hus let $N_{j}(t)$ be the number of accidents of type $i$ which occur up to time $t$. $N(t)=N_{1}(t)+\ldots+N_{n}(t)$ is the totai number of 
accidents of a!l types which occur at the plant up tn time $t$. Writing $M(t)=E N(t)$ and $M_{i}(t)=E N_{i}(t)$ then of course $M(t)=M_{j}(t)+\ldots+M_{n}(t)$. He define the above to be a system of rare evenis if for eacit $t$

$$
\text { i) } \lim _{n \rightarrow \infty} \max _{i=1, \ldots, n} P\left[N_{i}(t)=0\right]=1 \text {, }
$$

and

$$
\text { ii) } \lim _{n \rightarrow \infty} \prod_{i=1}^{n} P\left[N_{i}(t ; \leq 1]=1\right. \text {, }
$$

We interpret i) as saying that an accident of any given type is unlikely; ii) says that two accidents of any one type are unlikely. The relation ii) is usually stated in the equivalent form

$$
\text { ii') } \lim _{n \rightarrow \infty} \sum_{i=1}^{n} P\left(N_{i}(t) \geq 2\right)=0 \text {; }
$$

ii') has the consequence that $\lim M_{i}(t)=P\left[N_{i}(t)=1\right]$ anc

$$
\lim _{n \rightarrow \infty} M(t)=\lim _{n \rightarrow \infty} \sum_{i=1}^{n} P\left[N_{i}(t)=i\right] .
$$

With this background of language, the Theorem of Grigelionis, see Gnedenko (1967, p. 428), states

Theorem. For a system of rare events, the necessary and sufficient condition that tiie summary process $N(t)$ converges to a Poisson process with $\operatorname{EN}(t)=\Lambda(t)$ is that for any fixed $s$ and $t(s<t)$

$$
\lim _{n \rightarrow \infty} \sum_{i=1}^{n} P\left[N_{i}(t)-N_{i}(s)=1\right]=\Lambda(t)-\Lambda(s) .
$$

Thus the summary process of accidents is approximately Poisson even though the various types may not be. One consequence is that the probability of no accident in time $t$ is approximately

$$
P\{1(t)=0)=\exp \left\{\sum_{1 \leq 1}^{n} P\left(N_{f}(t)=1\right)\right\}
$$




\section{CONSEQUENCES, COMPARISON OF RISKS}

The actuarial theory of risk, see Beard, Pentıkäinen and Pesonen (1969), loviks at things from the point of view $s !$ an insurance company. Here the consequences of the unwanted events are clear; they are the monetary claims which must be paid. The object of the theory is to determine premiums that will avoid depletion of the insurance pool and consequent ruin of the company by a run of bad luck.

In statistical decision theory, loss is monetary or utility loss and risk is expected ioss. Business experts have their problems of risk and perhaps, with their profit motive they can assign values to the consequences of events and push through their cost-benefit analyses.

The idea of cost-benefit analysis is that cost will rise (roughly) linearly with activity level but, while initial benefit is easy to obtain, equal increments of benefit will require continually greater effort. Hence cost will rise to meet benefit and that is the level of activity which should be undertaken. Risk-benefit analysis then attempis to carry this accounting techni ue over into the social area to determine the amount of technological activity of a certain kind at which risk is balanced by benefit.

Rut it is not ciear to what extent this Bernoulli-Von NeumannMorgenstern-Wald theory can or should be carried over to things like reactor safety. It seems that reactor safety is in large part concerned with potential negative consequences to society. These negative consequences will be measured in terms of premature deaths, accidents, and compromised quality of living, as well as monetary loss. Devices like placing the cost of a fatality at 6000 man days of labor are hard to 
defend. It is inlikely that we will be able to "price" many of the consequences which will be our primary interest.

Demographers have been studying problems of risk for many jears. Perhaps the busiriess concept has too often been employed where the denographic idea is more appropriate.

For the consequence of iminediate fatalities periaps risk shculd be measured relative to a mortality table such as that of Jable 1, taken from the World Almanac (1978). The principle mathematical entity here is the force of mortality which interestingly is sometimes called the intensity of risk of dying.

Table 1. Average Lifetime in U.S., 1976

Source: Division of Vital Statistics, Public Health Service

\begin{tabular}{|c|c|c|c|c|c|}
\hline Age Interval & $\begin{array}{l}\text { Number } \\
\text { Living } \\
\end{array}$ & $\begin{array}{l}\text { Avg. Life } \\
\text { Expect. } 2\end{array}$ & Age !nterval & $\begin{array}{l}\text { Number } \\
\text { Living }\end{array}$ & $\begin{array}{l}\text { Avg. Life } \\
\text { Expect. } 2 \\
\end{array}$ \\
\hline $\begin{array}{l}0-1 \\
1-5 \\
5-10 \\
10-15 \\
15-20 \\
20-25 \\
25-30 \\
30-35 \\
35-40 \\
10-45\end{array}$ & $\begin{array}{r}100,000 \\
98,488 \\
98,207 \\
98,033 \\
97,865 \\
97,391 \\
96,747 \\
96,147 \\
95,452 \\
94,522\end{array}$ & $\begin{array}{l}72.8 \\
72.9 \\
69.1 \\
64.2 \\
59.3 \\
54.6 \\
44.9 \\
45.2 \\
40.5 \\
35.9\end{array}$ & $\begin{array}{l}45-50 \\
50-55 \\
55-60 \\
60-65 \\
65-70 \\
70-75 \\
75-80 \\
80-85 \\
85 \text { and up }\end{array}$ & $\begin{array}{l}93,043 \\
90,747 \\
87,310 \\
82,282 \\
75,084 \\
66,114 \\
54,060 \\
39,540 \\
25,029\end{array}$ & $\begin{array}{l}37.4 \\
27.2 \\
23.1 \\
19.4 \\
16.0 \\
12.8 \\
10.1 \\
7.9 \\
6.0\end{array}$ \\
\hline
\end{tabular}

(1) Of 100,000 born alive, number living at beginning of age interval

(2) Average number of years of iffe remaining at beginning of age interval.

It may be of some value to distinglifsh a subtopic of risk analysis which we might call safety analysis. Here the unwanted events are called accidents and the negative consequences are premature death, injury, pain, etc. The mortality of mankind imposes a constraint on any realistic 
safety analysis. Perhaps it is the uipleasantness of this condition which causes this obviuus fact to be omitted from many safety anaiyses. Here we see that the quetation from : anklin is precise?y to the point.: The question is not whether to gamble, it is ?rm to gamble. Tha statistical discipline of competing risks may be a p.oper framework within wich to analyse this subset of problems.

Competing risk theory hypothesizes a number of risks (diseases, accidents, etc.) which compete for the lives of individuals. For each individual one of there diseases will "win out" and the individial will die from that risk. The theory then attempts to predict the consequence of removing or adding a risk. For example, what would be the effect on life expectancy if a cure for smallpox were found? Similarly we might ask, What would be the effect on life expectancy if one hundred atomic power plants were added to the community?

Let $Y$, the random lifetime of an individual from the community, have distribution $G(v)$ and density $g(y)$. The force of mortality is defined as $h(y)=g(Y) / \bar{G}(y)$ where $\bar{G}(y)=1-G(y)$; it has the following interpretation: : $f$ we know that an individual has reached age $y$ then the probability that he will survive for an additional short time, $\Delta$, is approximately $h(y) \Delta$.

If a new independent risk is introduced and the individual dies from either the new risk or one of the old, depending on which occurs earlier, then it is a theorem that the new risk force of mortality will add to that of the risks already present. Postulating that the new risk force of mortality is constant over age, say $\lambda$, then the probability that an individual will eventually die from the new risk rather than one 
to witich he was previously exposed is

$$
\begin{aligned}
\pi(\lambda) & =1-\int_{0}^{\infty} \exp (-\lambda y) g(y) d y \\
& =\lambda 1+-\lambda^{-} x / 2+c\left(\lambda^{2}\right)
\end{aligned}
$$

Where $\mu$ and $k$ are the first and sicond moments of $g$ and $o\left(\lambda^{2}\right)$ is a remainder term which if dividec by $\lambda^{2}$ goes to zero.

Introduction of an additional risk will decrease the expected life length of an exposed individual. With the new risk included, expected life length will be

$$
\begin{aligned}
\int_{0}^{\infty} \exp (-\lambda y) \bar{G}(y) d y & =\lambda^{-1} \pi(\lambda) \\
& =\mu-\lambda k / 2+o(\lambda) .
\end{aligned}
$$

Thus the reduction in expected life length will be approximately $\lambda_{k} / 2$.

For example, from Table 1, the 1976 Unitied States experience is that $\mu=72.8$ and $\kappa=5625.41$. The latter value follows from Table 1 assuming average individuals to die at interval midpoints and using the fact that a person reaching eighty-five has average additional life expectancy of six years. From HASH 1400, if 100 nuclear power plants are introduced and nuclear accident were the only risk, the individual early fatality probability per year is $2 \times 10^{-10}$.

Thus the probability of fatality from nuclear accident rather than some already existing risk would be approximately $72.8 \times 2 \times 10^{-10}$ or $10^{-8}$ The reduction in expected life length will be approximately $2 \times 10^{-10} \times$ $5625.41 / 2=6 \times 10^{-7}$ years or about 18 seconds. Of course, these morta ity figures are no more accurate than their input and WASH 1400 is controversial; but here we are 11 lustrating a method of presentation which would be applifable to any iuch study. For instance, if we change the 
$2 \times 10^{-10}$ figure to $10^{-6}$ then $\pi^{\prime}\left(10^{-6}\right)=7 \times 10^{-5}$ and the rediction in life expectancy due to introducing the new risk is about one day.

As in MASH 1400 let's place these figures in context by considering motor vehirle fatalities. Again we bas a our computations on the 1976 United States experience as given in the (1978) World .i imanac. From Table 2-(2) it appears that the new driver ages of 15-24 are pal-icularly pron to fatal motor vehicle accidents; hence an assumption of constant risk will not be yalid and the previously explained methods will not be applicable. Table 2 outlines a method which is applicable for calculating what the age distribution of fatalities would te if the risk due to motor vehicle accidents could be eliminated. The 1976 proportion of deaths due to motor vehicle accidents was $45,800,1,912,000=0.024$. In Table 2 "all" risks refers to all nonnuclear risks and "other" refers to risks excluding nuclear as well as motor vehicle risks. The life expectancy would rise to 73.6 years if metor vehicle risks were eliminated. This follows from Table 2-(6) again assuming that average individuals die at midpoints.

Table 3 summarizes the competing risk presentation of the Reactor Safety Stucy in so far as early fatality risk is concerned. Looking at life expectancy seems to be an effective way of quantifying this kind of risk. The above computations are however deficient in that many fatalities due to nuclear accident will not be inmediate. Further work is needed in order to quantify the risk of these delayad fatalities. 
Table 2. Motor Vehicle Fatality Risk vs All Other

\begin{tabular}{|c|c|c|c|c|c|}
\hline \multirow{2}{*}{$\begin{array}{c}\text { age } \\
\text { interval }\end{array}$} & \multirow{2}{*}{$\begin{array}{c}\text { survival } \\
\text { probabilities, } \\
\text { all risks }\end{array}$} & \multirow{2}{*}{$\begin{array}{l}\text { fatality } \\
\text { probabilities, } \\
\text { moter vehicle }\end{array}$} & \multirow{2}{*}{\multicolumn{2}{|c|}{ force of mortality }} & \\
\hline & & & & & \\
\hline
\end{tabular}
(1)
(2)
(3)
(4)
(5)
(6)

$\begin{array}{lr}\text { under } 5 & 1.000 \\ 5-14 & .982 \\ 15-24 & .979 \\ 25-34 & .967 \\ 35-44 & .955 \\ 45-54 & .930 \\ 55-64 & .873 \\ 65-74 & .751 \\ 75-84 & .541 \\ 85 \text { and over } & .250\end{array}$

.034
.072
.342
.167
.094
.089
.078
.066
.058
.000

.0179
.0035
.0114
.0134
.0252
.0616
.1400
.2800
.5370
1.0000

.0008
.0018
.0084
.0042
.0024
.0023
.0021
.0021
.0026
.0000

.0171

(1) Proportion surviving to beginning of interval for all risks.

(2) Motor vehicle accident death proportions by age.

(3) Force of mortality for all risks; calculated from (1).

(4) $=0.024 \times(2) /(1)$.

(5) $=(3)-(4)$.

(6) Fatality probabilities by age if motor vehicle accidental deaths were eliminated; calculated from (5). 
Table 3. Probabilities of Eventual Dedth from Different Competing Risks

\begin{tabular}{|c|c|c|c|c|}
\hline & \multirow{2}{*}{$\begin{array}{l}\text { All Risks } \\
\text { but Nuclear }\end{array}$} & \multirow{2}{*}{$\begin{array}{l}\text { All Risks but } \\
\text { Muclear and } \\
\text { Motor Vehicle }\end{array}$} & \multicolumn{2}{|c|}{ All Risks } \\
\hline & & & $\lambda=2 \times 10^{-10}$ & $\lambda=10^{-6}$ \\
\hline Other & 0.976 & 1.0 & 0.976 & 0.976 \\
\hline Motor Vehicle & 0.024 & 0.0 & $\mathbf{0 . 0 2 4}$ & 0.024 \\
\hline Nuclear & 0.0 & 0.0 & $10^{-8}$ & $7 \times 10^{-5}$ \\
\hline $\begin{array}{l}\text { Life Expectancy } \\
\text { (Years) }\end{array}$ & 72.8 & 73.6 & $72.8(-18 \mathrm{sec})$ & $72.8(-1$ day $)$ \\
\hline
\end{tabular}


REFERENCES

R. E. Beard, T. Pentikăinen and E. Pesonen, 1969, Risk Theory, Methuen.

H. W. Lewis et al., 1978, "Risk assessment review group report to the U.S. Nuclear Reguiatory Commission," U.S. Huciear Regulatory Commission, NURE6/CR-0400.

6. J. Lieberman, "Fault tree analysis as an example of risk," Energy and the Enoironent a Risk-Benefit Approach, Eds. H. Ashley, R. Rudman and C. Mnipple, Pergamon Press.

N. R. Mann, R. E. Schafer and N. D. Singpunwalla, 1974, Methodis for Statistical Analysis of Reliability and Life Data, Niley.

N. C. Rasmussen, 1974, "The AEC study on the estimation of risks to the public from potential accidents in nuclear power plants," Nuclear Safety, 15.375-383.

H. D. Rowe, 1977, An Anatomy of Risk, Hiley.

M. Spiegelman, 1976, Introduction to Demography, Revised Edition, Harvard.

C. Starr, R. Rudman and C. Whipple, 1976, "Philosophical basis for risk analysis," Annual Review of Energy, 629-6א2.

W. A. Thompson Jr. and R. E. Funderlic, 1979, "A comparison of doseresponse mode1s," to appear.

WASH-1400, Reactor Safety Study, 1975, "An assessment of accident risks in U.S. commerctal nuclear power plants," (NUREG-75/014). United States Nuclear Regulatory Comission.

Webster, 1976, Third New Intermational Dictionary, 6 \& C. Merriam Co.

World AZmanas and Book of Eacts, 1978, Newspaper Enterprise Assoctation Inc., New York. 